\title{
Influence of cross-linking agent on the hydrophobicity of microencapsulated ammonium polyphosphate with tung oil phenolic resin
}

\author{
Weiying $\mathrm{GaO}^{1,2,3, \mathrm{a}}$, Shujun Wang ${ }^{1,2, \mathrm{~b}}$, Fanbin Meng ${ }^{1,2}$, Huanqing $\mathrm{Ma}^{1,2}$ \\ and Yuanhao Wang ${ }^{4}$, \\ ${ }^{1}$ State key laboratory of heavy oil processing, China University of Petroleum (Beijing), Beijing \\ 102249, China; \\ ${ }^{2}$ College of Science, China University of Petroleum (Beijing), Beijing 102249, China; \\ ${ }^{3}$ Department of Science and Technology, Chinese People's Armed Police Force Academy, Langfang \\ 065000,China; \\ ${ }^{4}$ Faculty of Science and Technology, Technological and Higher Education Institute of Hong Kong, \\ New Territories, Hong Kong. \\ agaoweiy12@sohu.com, bbjwsjbj@sina.com
}

Keywords: Ammonium polyphosphate; microencapsulation; tung oil phenolic resin; cross-linking agent, hydrophobicity.

\begin{abstract}
Ammonium polyphosphate (APP) was encapsulated with different cross-linked structure tung oil phenolic resin(TPF) by in situ polymerization and it was performed that a study on the influence of the cross-linking agent type on the hydrophobicity of the APP microcapsules.The chemical and physical features of APP microcapsules were characterized by Fourier transform infrared(FTIR), X-ray photoelectron spectroscopy(XPS). The hydrophobicity was assessed by the water contact angle(WCA). The results showed that the APP microcapsules with different cross-linked structure TPF shell had been achieved successfully and the cross-linking agent type had important influence on the hydrophobicity of APP microcapsules.
\end{abstract}

\section{Introduction}

Because of its low smoke, low-toxicity and less corrosive substances during its combustion, ammonium polyphosphate [ $\left(\mathrm{NH}_{4}\right)_{n+2} \mathrm{P}_{\mathrm{n}} \mathrm{O}_{3 \mathrm{n}+1}$ ] is widely used as flame retardant in the fields of coating industry, plastics, wood, rubber or as extinguisher in the fields of forest fire, coal field fire. When exposed to an external heat flux, APP would play a role as acid source to react with carbon source and gas source to give off inert gas, e.g. $\mathrm{N}_{2}, \mathrm{NH}_{3}, \mathrm{CO}_{2}$ and form a intumescent carbonaceous char layer as a kind of physical barrier to inhibit the transfer of heat and diffusion of inflammable gases ${ }^{[1-4]}$. Unfortunately, The fire retardancy of APP is not permanent due to its weak water resistance and poor compatibility in polymers. Microencapsulation technique is a good choice to overcome above problems. In previous study by Wu et al., microcapsules containing APP with various shells, such as melamine-formaldehyde, nylon-6, ureaformaldehyde, epoxy resin and gel-silica, were reported $^{[5-10]}$. To the best of our knowledge, microcapsules containing APP with tung oil-modified phenolic resin shell has not yet been reported.

The main content of tung oil is $\alpha$-Eleostearate.The tung oil phenolic resin (TPF) is a modified phenolic resin by introducing $\alpha$-Eleostearate into the main chain of a phenolic resin(PF). The flexibility of PF has been improved and it is more hydrophobic and easily compatible with polymer due to the existence of $\alpha$-Eleostearate ${ }^{[11]}$. Based on above mentioned advantages, a novel type of APP microcencapsulated with TPF (TPFAPP) was prepared and a study on the influence of the cross-linking agent type on the hydrophobicity of APP microcapsules was performed in this paper. 


\section{Experimental}

\subsection{Materials}

APP( an average degree of polymerization $200<\mathrm{n}<400$ )was supplied by Wuhan Hongxinkang Fine Chemical Corp.,Ltd, China. TPF was purchased from Anhui Tianyu High Temperature Resin Material Corp.,Ltd. Hexamethylene Tetramine( HMTA) ,ethylene diamine(EDTA), ethanol and chloroform used were reagent grade.

\subsection{Preparation of microcapsules}

A $250 \mathrm{ml}$ three-necked round bottom flask was equipped with a mechanical stirrer. Then, 8.6g $41.5 \%$ TPF ethanol solution and $63.4 \mathrm{~g}$ ethanol were poured into the flask and the mixture was stirred for $5 \mathrm{~min}$ at room temperature. Then, $10 \mathrm{~g}$ APP, $1 \mathrm{~g}$ OP-10, and $0.5 \mathrm{~g}$ dimethylsilicon oil were added and stirred for $15 \mathrm{~min}$. After that $16 \mathrm{~g}$ 4.54\% cross-linking agent ethanol solution was added dropwise to the mixture, keeping at the same temperature for $1 \mathrm{~h}$. Finally the mixture were filtered, washed with ethanol, and dried at $60{ }^{\circ} \mathrm{C}$ to achieve the final microcapsulated APP powders. In order to further investigate the influence of different cross-linking agent on the hydrophobicity of microcapsules, some samples were cured at $160^{\circ} \mathrm{C}$ for $3 \mathrm{~h}$.

APP microcapsules with HTMA cross-linking TPF shell was represented by HTPFAPP, APP microcapsules with EDTA cross-linking TPF shell was represented by ETPFAPP.The weight ratio of TPF to cross-linking agent was 1:0.2.

\subsection{Measurements}

\subsubsection{Fourier transforms infrared analysis}

FTIR spectra were obtained on a JASCO480 spectrometer (German Bruker) with thin $\mathrm{KBr}$ as the sample holder. Transition mode was used and the wave number range from 4000 to $500 \mathrm{~cm}^{-\square 1}$.

\subsubsection{X-ray photoelectron spectroscopy}

The XPS spectra were obtained on a K-Aepna spectrometer (Thermo Fisher Scientific,USA) with $\mathrm{Al} k \alpha$ excitation radiation $(h v=1253.6 \mathrm{eV})$ in ultrahigh vacuum conditions.

\subsection{3 hydrophobicity analysis}

The hydrophobicity of samples was characterized by contact angle of the powders with deionized water. The powder samples were first pressed into wafers at $15 \mathrm{MPa}$ and then measured using JC2000D contact angle analyzer (Shanghai Zhongchen Digital Technology Equipment Co., Ltd. China).

\section{Results and discussion}

\subsection{Chemical characterization}

The FTIR spectra of APP,HTPFAPP and ETPFAPP are presented in Fig.1. As seen in Fig.1(a), the important characteristic absorption bands of APP were as follows: $3200 \mathrm{~cm}^{-\square 1}$ (N-H symmetric stretching vibration), $1692 \mathrm{~cm}^{-\square 1}\left(\mathrm{~N}-\mathrm{H}\right.$ bending vibration), $1239 \mathrm{~cm}^{-\square 1}(\mathrm{P}=\mathrm{O}), 1065 \mathrm{~cm}^{-\square 1}(\mathrm{P}-\mathrm{O}$ symmetric stretching vibration), $871 \mathrm{~cm}^{-\square 1}$ (P-O asymmetric stretching vibration), $1020 \mathrm{~cm}^{-\square 1}\left(\mathrm{PO}_{2}\right.$ and $\mathrm{PO}_{3}$ ) and $800 \mathrm{~cm}^{-\square 1}$ (P-O-P). After being coated by TPF,the spectra changed,as seen in Fig.1(b) and Fig.1(c). The intensities of absorption peak at $3400 \mathrm{~cm}^{-\square 1}$ and $1636 \mathrm{~cm}^{-\square 1}$ increased due to introducing TPF groups:O-H and $\mathrm{C}=\mathrm{C}$, indicating the existence of TPF in the structure of TPFAPP. In addition, the absorption peak of ETPFAPP were more stronger than that of HTPFAPP,which showed that the amount of precipitated TPF due to EDTA cross-linking(ETPF) was more than that of precipitated TPF due to HTMA cross-linking(HTPF).

It can be seen from Fig.2(a) that the peak at $134.24 \mathrm{eV}, 400.26 \mathrm{eV}, 285.04 \mathrm{eV}$ and $532.06 \mathrm{eV}$ are assigned to $\mathrm{P}_{2 \mathrm{p}}, \mathrm{N}_{1 \mathrm{~s}}, \mathrm{C}_{1 \mathrm{~s}}, \mathrm{O}_{1 \mathrm{~s}}$ of APP. After microencapsulation, the intensities of $\mathrm{P}_{2 \mathrm{p}}, \mathrm{N}_{1 \mathrm{~s}}$ and $\mathrm{O}_{1 \mathrm{~s}}$ peaks greatly decreased and the intensities of the $C_{1 s}$ peaks, centered at $290.08 \mathrm{eV}$, increased sharply,as seen in Fig.2(b) and Fig.2(c). The above changes indicated that APP was well coated by TPF. Meanwhile the intensities of $\mathrm{P}_{2 \mathrm{p}}$ and $\mathrm{N}_{1 \mathrm{~s}}$ peaks of ETPFAPP were weaker than that of HTPFAPP, indicating that APP was better coated by ETPF, which was in accordance with the data of FTIR. 


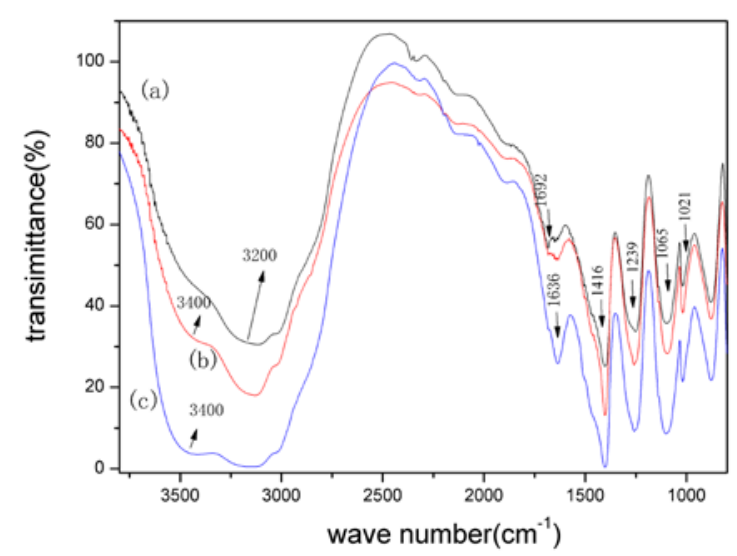

Fig.1 FTIR spectra of (a) APP,(b) HTPFAPP,(c) ETPFAPP
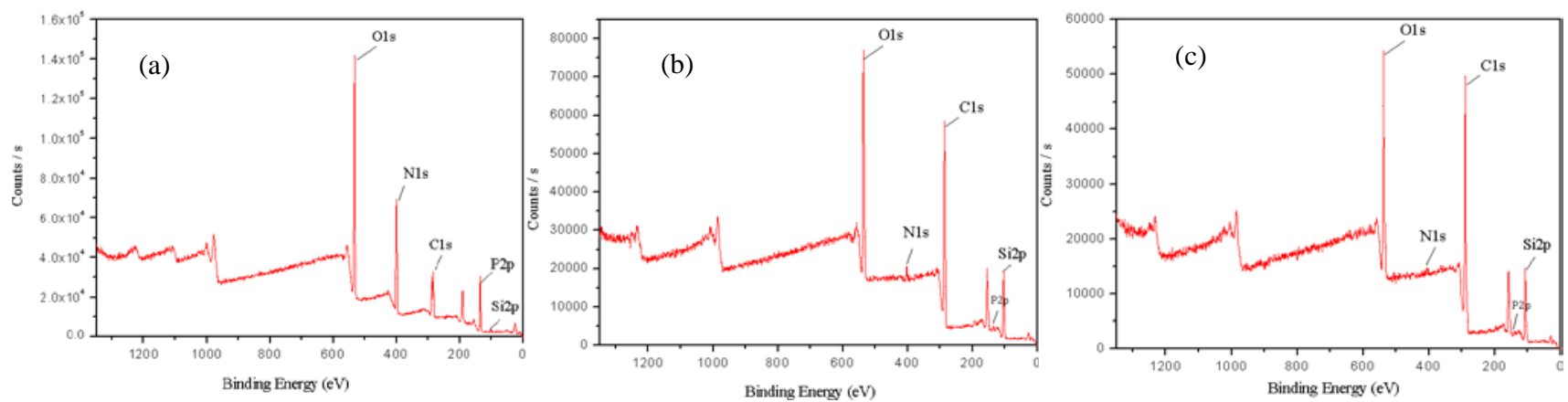

Fig.2. XPS spectra of (a) APP, (b) HTPFAPP,(c) ETPFAPP

\subsection{Hydrophobicity of APP microencapsules}

The hydrophobicity was assessed by the water contact angle(WCA).The larger the WCA is, the better the hydrophobicity is. The WCA photographs of APP and its microencapsules are shown in Fig.3.It can be seen from Fig.3(a) that the WCA of original APP was $23^{\circ}$, showing that APP was very hydrophilic. After microencapsulation, the WCAs of HTPFAPP and ETPFAPP all sharply increased, improving the hydrophobicity of APP. However, the WCA of HTPFAPP was $80^{\circ}$, lower than that of ETPFAPP $\left(86^{\circ}\right)$,as seen in Fig.3(b) and Fig.3(c). It is mainly because the amount of precipitated ETPF was more than that of precipitated HTPF and APP was better coated by ETPF, which can be confirmed by the data of FTIR and XPS. But after heat curing, the WCAs of HTPFAPP and ETPFAPP increased to $104^{\circ}$ and $93^{\circ}$ respectively due to the removal of some hydroxyl and the formation of lipophilic groups,as seen in Fig.3(d) and Fig.3(e).The WCA of HTPFAPP increased by $24^{\circ}$, higher than $8^{\circ}$ by which the WCA of ETPFAPP increased, indicating that the structure of HTPF was differnt from that of ETPF and APP was better coated by HTPF or HTPF itself being more hydrophobic owing to thermo-crosslinking. The above results showed that the type of cross-linking agent had important influence on the hydrophobicity of APP microcapsules. The main reasons are as follows: Although the film-forming materials are all TPF, the mechanisms of TPF cross-linking are different due to different cross-linking agent,so different structure TPF will form, leading to different physical and chemical properties, which can be also confirmed by the powders color.From the visual perspective, HTPFAPP was a white powder, slightly red. ETPFAPP was a lighter yellow powder. After heat curing, HTPFAPP turned to orange and ETPFAPP turned to brown. 

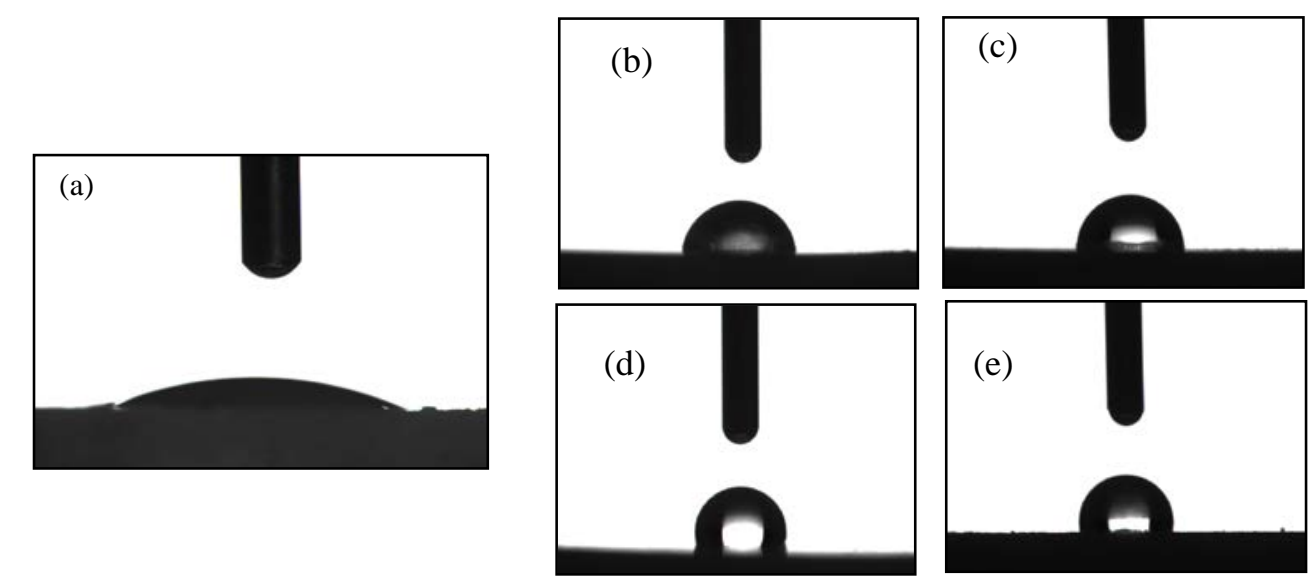

Fig.3 WCA photographs:(a) APP, (b) HTPFAPP, (c) ETPFAPP , (d) Heat cured HTPFAPP, (e) Heat cured ETPFAPP

\section{Conclusion}

In this paper, APP was encapsulated with different cross-linked structure tung oil phenolic resin by in situ polymerization and it was performed that a study on the influence of the cross-linking agent type on the hydrophobicity of APP microcapsules.The FTIR and XPS analysis showed that APP microcapsules with different cross-linked structure TPF shell had been achieved successfully and APP was better coated by ETPF before heat curing. The WCA data indicated that the type of cross-linking agent had important influence on the hydrophobicity of APP microcapsules.The hydrophobicity of ETPFAPP was better than that of HTPFAPP. However, HTPFAPP was more hydrophobic after heat curing.The main reason is that the mechanisms of TPF cross-linking are different due to different cross-linking agent,so different structure TPF will form, which lead to different physical and chemical properties.

\section{References}

[1]. Wu Q, Qu B. Synergistic effects of silicotungistic acid on intumescent flame-retardant polypropylene. Polym Degrad Stab. Vol.74(2001), p. 255-261.

[2]. Chen Y H, Liu Y, Wang Q et al. Performance of intumescent flame retardant master batch synthesized through twin-screw reactively extruding technology:effect of component ratio. Polym Degrad Stab. Vol.81(2003), p. 215-224.

[3]. Ni JX, Chen LJ, Zhao KM et al. Preparation of gel-silica/ammonium polyphosphate core-shell flame retardant and properties of polyurethane composites. Polym Adv Technol. Vol. 22(2011), p.1824-1831.

[4].D. Saihi, I. Vroman, S. Giraud et al. Microencapsulation of ammonium phosphate with a polyurethane shell, Part II. Interfacial polymerization technique. Reac Func Polym. Vol.66( 2006),

p. $1118-1125$.

[5]. Wu K, Zhang YK, Hu WG et al. Influence of ammonium polyphosphate microencapsulation on flame retardancy, thermal degradation and crystal structure of polypropylene composite.Composites Science and Technology. Vol. 81(2013), p.17-23.

[6].Liu MF, Liu Y,Wang Q. Flame-retarded poly(propylene) with melamine phosphate and pentaerythritol/polyurethane composite charring agent.Macromol Mater Eng.Vol.292(2007),No.2,

p. 206-13.

[7].Wu K, Song L,Wang ZZ et al. Preparation and characterization of double shell microencapsulated ammonium polyphosphate and its flame retardance in polypropylene. J Polym Res. Vol.16, No.3 (2009), p.283-294.

[8].Wu K, Song L, Wang ZZ, Hu Y. Microencapsulation of ammonium polyphosphate with 
urea-melamine-formaldehyde resin and its flame retardance in polypropylene. Polym Adv Technol. Vol.19(2008),No.12, p.1914-1921.

[9].Giraud S., Salaüna F., Bedek G. et al. Influence of chemical shell structure on the thermal properties of microcapsules containing a flame retardant agent. Polym Degrad Stab Vol.95( 2010), p.315-319.

[10]. Chen XL, Jiao CM, Zhang J. Microencapsulation of ammonium polyphosphate with hydroxyl silicone oil and its flame retardance in thermoplastic polyurethane. J Therm Anal Calorim. Vol.104(2011), p.1037-1043.

[11]. Yu G, LuPS, Wang Y, et al. Study on phenol-formaldehyde resins modified with tung oil( $\quad I)$ - Synthesis, Mechanism and Characterization. Chemistry and Industry of Forest an Products, Vol.14(1993), p. 23-31. 